\title{
Incidence Des Representations Sociales Sur La Durabilite Sociale De L'amenagement Des Forets Classees Des Monts Kouffe Et De Wari-Maro Au Benin
}

\author{
BAGUIRI Oumêmath \\ TOHON Sètongninougbo Hermann Eric \\ TINGBE AZALOU Albert
}

Laboratoire d'Analyse des Dynamiques Socio-anthropologiques et d'Expertises pour le Développement, Université d'Abomey-Calavi (UAC), Département de Sociologie-Anthropologie (DS-A), Faculté des Sciences Humaines et Sociales (FASHS)

\section{SOUAND Tahi}

Laboratoire de Biomathématiques et d'Estimations Forestières, Faculté des Sciences Agronomiques, Université d'Abomey-Calavi (UAC), Bénin

Doi:10.19044/esj.2020.v16n28p150 URL:http://dx.doi.org/10.19044/esj.2020.v16n28p150 Submitted: 7 September 2020 Accepted: 28 September $2020 \quad$ Published: 31 October 2020 Copyright 2020 Oumêmath et al. Distributed under Creative Commons CC-BY 4.0 OPEN ACCESS

\section{Résumé}

Les gestionnaires des ressources naturelles, ne voient généralement de la forêt que la production du bois en occultant les dimensions symboliques de ces espaces. Les communautés vivant aux alentours de ces espaces forestiers ont un lien étroit avec les forêts dont découlent des représentations spécifiques qui influencent la gestion durable des forêts. Cette recherche a pour objectif d'identifier les représentations sociales et leur incidence sur la durabilité sociale de l'aménagement des forêts classées des Monts Kouffé et de WariMaro. L'approche méthodologique utilisée est mixte. Des questionnaires ont été administrés et des entretiens individuels et de groupe menés auprès de 224 acteurs choisis suivant un échantillonnage aléatoire stratifié et un choix raisonné dans une dizaine de villages riverains des deux forêts classées. Les résultats montrent que les représentations sociales de la forêt sont multiples. Les communautés locales se représentent la forêt classée comme un bien naturel que l'Etat leur a arraché, ce qui a un impact sur les pratiques et usages qu'elles en ont et sur la durabilité sociale de l'aménagement forestier. Ensuite, les communautés riveraines se représentent la forêt comme un espace destiné à leur permettre de satisfaire leurs besoins. Elles utilisent donc les ressources forestières sans aucune considération pour le plan d'aménagement en vigueur dans la mesure où pour ces populations, la forêt 
appartient à Dieu. Etant également considérée comme une zone d'insécurité, l'utilité de la forêt est souvent remise en cause.

Mots clés: Représentations sociales, recherche mixte, durabilité sociale, Monts Kouffé et Wari-Maro, Bénin. 


\title{
Impact of Social Representations On the Social Sustainability of the Management of the Classified Forests of Monts Kouffe and Wari-Maro in Benin
}

\author{
BAGUIRI Oumêmath \\ TOHON Sètongninougbo Hermann Eric \\ TINGBE AZALOU Albert
}

Laboratoire d'Analyse des Dynamiques Socio-anthropologiques et d'Expertises pour le Développement, Université d'Abomey-Calavi (UAC), Département de Sociologie-Anthropologie (DS-A), Faculté des Sciences Humaines et Sociales (FASHS)

\section{SOUAND Tahi}

Laboratoire de Biomathématiques et d'Estimations Forestières, Faculté des Sciences Agronomiques, Université d'Abomey-Calavi (UAC), Bénin

\begin{abstract}
The managers of natural resources, generally see only the forest as the production of wood by obscuring the symbolic dimensions of these spaces. The communities living around these forest areas have a close link with the forests from which arise specific representations that influence the sustainable management of forests. This research aims to identify social representations and their impact on the social sustainability of the management of the classified forests of the Monts Kouffé and Wari-Maro. The methodological approach used is mixed. Questionnaires were administered and individual and group interviews carried out with 224 actors chosen according to a stratified random sampling and a reasoned choice in ten villages bordering the two classified forests. The results show that the social representations of the forest are multiple. Local communities see the classified forest as a natural asset that the state has taken from them, which has an impact on the practices and uses they have of it and on the social sustainability of forest management. Next, the riparian communities see the forest as a space intended to enable them to meet their needs. They therefore use forest resources without any consideration for the current management plan, since for these populations the forest belongs to God. Being also considered an area of insecurity, the usefulness of the forest is often questioned.
\end{abstract}

Keywords: Social representations, mixed research, social sustainability, Monts Kouffé and Wari-Maro, Benin. 


\section{Introduction}

La gestion des forêts classées béninoises est fondée sur une approche participative depuis l'adoption de la loi no 93-009 du 2 juillet 1993 portant régime des forêts en République du Bénin et de son décret d'application datant de 1996. L'article 40 de cette loi accorde une bonne place aux communautés locales dans cette gestion dont les clauses doivent être définies dans un plan d'aménagement. Les forêts classées des Monts Kouffé et de Wari-Maro ont bénéficié d'un plan d'aménagement élaboré grâce au concours du Projet d'Aménagement des Massifs Forestiers d'Agoua, des Monts Kouffé et de Wari-Maro (PAMF). Une décennie après l'élaboration de ce plan, la gestion participative qui devait conduire à une gestion durable des forêts n'a pas été concluante. Dans leur recherche sur les forêts dans la commune de Bassila, Gbedahi et al (2019) sont parvenus à la même conclusion et ont suggéré qu'il soit mis en place un système de suivi des activités d'aménagement après la fin des projets pour la gestion durable des forêts. Il se pose un problème de la non-durabilité sociale qui met en jeu les comportements des acteurs vis-à-vis des massifs forestiers d'Agoua, des Monts Kouffé et de Wari-Maro.

La question des pratiques sociales n'est pas souvent isolée de celle de leurs représentations sociales. En effet, les pratiques des individus vis-à-vis de leur environnement sont dictés par les représentations qu'ils en ont (Auclair, 1996 ; Diop, 2012 ; Ouassa Kouaro, 2014). Dans cette perspective, Caillaud (2010) pense qu'il existe une dichotomie entre visible/invisible, riche/pauvre qui viennent structurer les représentations des populations et par lesquelles les pratiques écologiques acquièrent des significations multiples. Dans le même ordre d'idées Weber (1995) estime que les représentations sociales définissent généralement l'environnement comme l'envers de la nature. Ainsi, la nature est propre, l'environnement est sale d'où les expressions "équilibres de la nature »et "déséquilibres environnementaux ». Les représentations des communautés sont donc conditionnées par les conditions dans lesquelles elles sont. Navarro (2009) explique les polémiques suscitées par l'utilisation des ressources naturelles, les responsabilités liées à leur gestion et les actions de conservation mises en œuvre, naissent de la diversité des " conceptions » et des usages. En effet, certains les considèrent comme un "bien commun » de l'humanité et d'autres comme un "bien économique » afin d'être plus efficace dans sa gestion. Il y a donc une différence de conception. Les ressources naturelles constituent un «besoin » humain et pour d'autres un «droit» humain.

Les pratiques et usages des communautés rurales sont relatifs aux activités qu'elles mènent en forêt, en bordure de forêt et dans le terroir riverain. Il s'agit des activités économiques mais aussi religieuses. Les 
incursions dans l'espace forestier doté d'un plan d'aménagement à peine respecté sont certainement le fait des représentations sociales que les communautés riveraines ont de ces forêts. En effet, ces représentations sont définies comme un savoir de sens commun car elles sont partagées par l'ensemble de la population ou une partie importante de cette population. Dans ce sens, Capozza et al. (1995) pense que le noyau central de la représentation est le binôme argent-pouvoir. La forêt tend de plus en plus à devenir un patrimoine mondial de l'humanité. C'est la question délicate de la patrimonialisation. Cependant, Abanda Ngono (2017) analyse la pluralité des enjeux autour de la forêt et surtout la diversité de ses représentations comme des éléments qui rendent problématique l'option de la patrimonialisation des forêts pour le compte de l'humanité dans des territoires où les populations en dépendent encore étroitement. Ainsi, les représentations sociales structurent le comportement des acteurs vis-à-vis de l'environnement. . Quelles sont alors les représentations sociales qu'ont les communautés riveraines des forêts classées des Monts Kouffé et de Wari-Maro et quelle est leur incidence sur la durabilité sociale de l'aménagement de ces espaces ?

Pour répondre à cette question, une hypothèse générale a été formulée : la durabilité sociale de l'aménagement des forêts classées des Monts Kouffé et de Wari-Maro dépend des représentations sociales des forêts. L'objectif de cette recherche est d'identifier les représentations sociales et leur incidence sur la durabilité sociale des Massifs forestiers d'Agoua, des Monts Kouffé et de Wari-Maro.

L'article s'articule autour des points suivants : présentation du cadre géographique de la recherche, de l'approche méthodologique, des résultats et de leur discussion.

\section{Cadre géographique de la recherche}

La forêt classée des Monts Kouffé est située entièrement dans la Commune de Bassila (Département de la Donga). Elle se trouve entre $1^{\circ} 40$ et $2^{\circ} 15$ de longitude est et entre $8^{\circ} 25$ et $8^{\circ} 50$ de latitude nord. Adjacente à la forêt des Monts Kouffé, la forêt classée de Wari-Maro est à cheval sur les Communes de Bassila et de Tchaourou, respectivement dans les Départements de la Donga et du Borgou. Géographiquement, elle se trouve entre $8^{\circ} 50^{\prime}$ et $9^{\circ} 10$ de latitude nord et $1^{\circ} 55$ et $2^{\circ} 25$ de longitude est (Figure $1)$.

La recherche a été effectuée dans une dizaine de villages riverains des deux forêts classées. Il s'agit de Bétérou, Sinahou, Samba, Wari-Maro, Agramarou, Alafiarou pour la zone riveraine de la forêt classée de WariMaro et des villages de Manigri Oké, Manigri Ikani, Biguina1, Biguina 2 et Biguina3 pour le territoire riverain de la forêt classée des Monts Kouffé. Dans la zone riveraine de la forêt classée de Wari-Maro, les groupes 
socioculturels dominants sont les Nagot et les Baatombu suivis des Peulhs et des Wama et Ditamari. Dans la zone riveraine des Monts Kouffé, les Nagot et les Lokpa sont dominants. Les Peulhs y sont également présents.

Il s'agit en majorité de peuples d'agriculteurs. Les Nagot sont spécialisés dans la chasse. L'exploitation forestière est une activité à laquelle s'adonnent de plus en plus les jeunes à la recherche de gain facile. Les pêcheurs sont aussi installés dans les villages riverains même si leurs activités sont souvent saisonnières en raison de ce que la plupart des cours d'eau sont temporaires. 


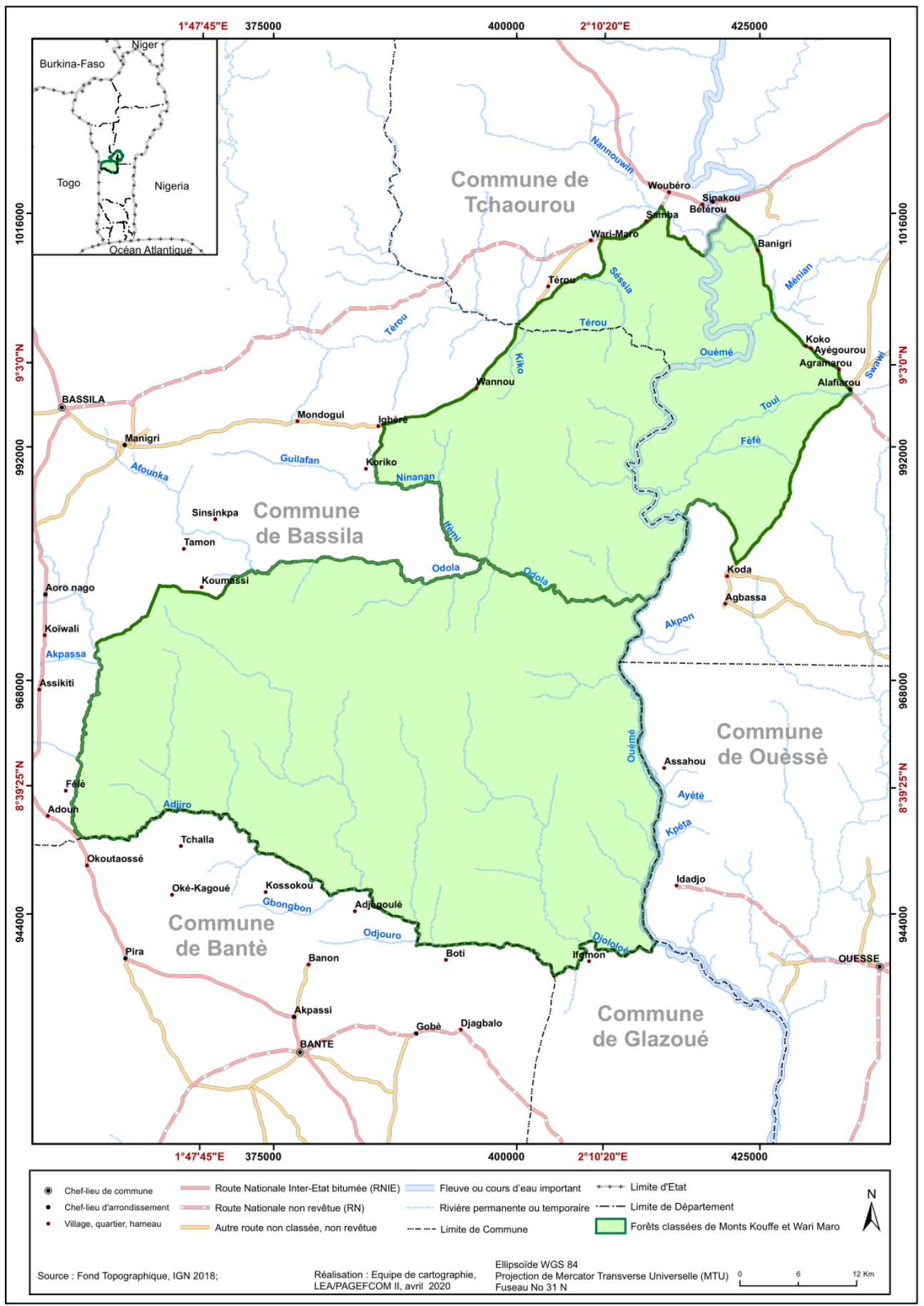

Figure 1: situation géographique des forêts classées des Monts Kouffé et de Wari-Maro Source : LEA/PAGEFCOM II (2020) 


\section{Approche méthodologique}

La présente recherche est inscrite dans la théorie des représentations sociales de Jodelet (1989). Elle est de nature mixte. Ainsi, l'entretien, l'enquête par questionnaire et le focus group sont les techniques utilisées pour la collecte des données.

\section{Techniques de collecte et d'analyse des données}

L'entretien a permis de comprendre en profondeur, les représentations sociales de la forêt qui sont favorables ou non à la durabilité sociale de l'aménagement forestier. Le questionnaire a été utilisé dans le but de quantifier l'information relative aux perceptions sociales afin de savoir si elles sont partagées. Cela permet de mettre alors en évidence, les représentations sociales de l'espace forestier car, sont considérées comme représentations sociales dans cette recherche, les perceptions qui ont recueilli au moins $50 \%$ de citation. Les focus group ont été utiles à l'appréhension des dynamiques au sein des groupes tels que les groupements de femmes et les comités de cogestion des forêts classées.

Les données recueillies ont été soumises à un dépouillement manuel. Celles qui sont issues du questionnaire ont été dépouillées grâce au tableur Excel et exportées vers logiciel $\mathrm{R}$ pour les analyses statistiques. Les données issues des entretiens et des focus group ont fait l'objet d'une transcription intégrale, d'un tri thématique et d'une analyse de contenu du discours des acteurs.

\section{Groupes cibles}

Les catégories d'acteurs sur lesquels a porté la recherche sont: les agriculteurs, les éleveurs, les chasseurs, les groupements de femmes, les exploitants forestiers, les membres des comités de cogestion, les forestiers, les Agents de Développement Local (ADL), les Techniciens d'Appui aux Organisations Paysannes (TAOP) et les autorités locales. Les critères d'inclusion sont : l'âge (un âge permettant de savoir qu'ils étaient au courant du processus d'aménagement des FCMK et FCWM c'est-à-dire au moins 40 ans), la participation aux activités du PAMF et la dépendance vis-à-vis de ces massifs forestiers. Au total 15 entretiens, 8 focus group et 122 questionnaires ont été nécessaires à l'appréhension de l'organisation sociale autour de l'aménagement forestier. Au total, 224 acteurs ont donc été choisis sur la base d'un échantillonnage aléatoire stratifié et par choix raisonné. 


\section{Résultats}

Pour identifier les représentations sociales de la forêt, il a fallu passer par les perceptions sociales de la forêt.

\section{Perceptions sociales des forêts classées des Monts Kouffé et de Wari- Maro}

Les rôles ou les caractéristiques les plus importants des forêts classées des Monts Kouffé et de Wari-Maro selon les populations riveraines interrogées sont : « la forêt classée appartient à l'Etat », « la forêt classée est une zone de conservation de la biodiversité », « la forêt classée, c'est la vie, c'est une réserve de bois (les arbres) », « la forêt classée est intégralement protégée et les ressources ne peuvent pas être exploitées par les populations locales », " la forêt classée est un refuge pour les prédateurs » et « la forêt classée contient des ressources naturelles qui vont améliorer les conditions de vie des populations locales ». Outre ces perceptions qui sont majoritaires dans les déclarations des répondants, plusieurs autres perceptions importantes ont été évoquées. Il s'agit des perceptions telles que «la forêt classée est une réserve de terre appartenant à Dieu », "la forêt classée est une zone d'insécurité », " la forêt classée est la demeure des dieux et des ancêtres », « la forêt classée représente des terres arrachées aux villageois/un gel des terres », " la forêt contient des ressources naturelles qui vont améliorer les conditions de vie des agents forestiers ", " la forêt classée contient des ressources naturelles qui vont améliorer les conditions de vie des exploitants étrangers ». Ces perceptions sont sous-tendues par des discours qu'il serait intéressant de relater à travers les représentations sociales de la forêt. Le tableau I présente les perceptions des acteurs locaux.

Tableau I: perceptions de la forêt classée par les acteurs locaux

\begin{tabular}{|l|l|}
\hline Perceptions & $\begin{array}{l}\text { Fréquences } \\
(\%)\end{array}$ \\
\hline La forêt appartient à l'Etat & 96 \\
\hline La forêt est une zone de conservation de la biodiversité & 95 \\
\hline La forêt représente la vie & 92 \\
\hline La forêt est une réserve de bois (les arbres) & 91 \\
\hline $\begin{array}{l}\text { La forêt est intégralement protégée et les ressources ne } \\
\text { peuvent pas être exploitées par les populations locales }\end{array}$ & 86 \\
\hline La forêt est un refuge pour les prédateurs & 85 \\
\hline $\begin{array}{l}\text { La forêt contient des ressources naturelles qui vont améliorer } \\
\text { les conditions de vie des populations locales }\end{array}$ & 85 \\
\hline La forêt est une réserve de terre appartenant à Dieu & 79 \\
\hline La forêt appartient à tout le monde & 75 \\
\hline
\end{tabular}




\begin{tabular}{|l|l|}
\hline La forêt est une zone d'insécurité & 75 \\
\hline La forêt est un espace boisé appartenant aux villages riverains & 74 \\
\hline La forêt est la demeure des dieux et des ancêtres & 62 \\
\hline $\begin{array}{l}\text { La forêt représente des terres arrachées aux villageois/un gel } \\
\text { des terres }\end{array}$ & 57 \\
\hline La forêt est calme & 40 \\
\hline La forêt est la brousse & 35 \\
\hline La forêt est une propriété du blanc & 34 \\
\hline $\begin{array}{l}\text { La forêt contient des ressources naturelles qui vont améliorer } \\
\text { les conditions de vie des agents forestiers }\end{array}$ & 23 \\
\hline $\begin{array}{l}\text { La forêt est contient des ressources naturelles qui vont } \\
\text { améliorer les conditions de vie des exploitants forestiers } \\
\text { étrangers }\end{array}$ & 14 \\
\hline La forêt est une zone de chasse & 13 \\
\hline La forêt est une réserve de plantes médicinales & 13 \\
\hline La forêt est un espace de conservation de la biodiversité & 08 \\
\hline La forêt est une zone de pâturage & 06 \\
\hline $\begin{array}{l}\text { La forêt est une ressource épuisable dont chacun doit prendre } \\
\text { sa part }\end{array}$ & 04 \\
\hline La forêt est une zone agricole & 04 \\
\hline La forêt est une zone d'exploitation forestière & 04 \\
\hline
\end{tabular}

Source : données de la recherche, 2018

\section{Représentations sociales de la forêt}

Les représentations sociales peuvent être considérées comme les discours qui sous-tendent les perceptions sociales de la forêt. $\mathrm{Si}$ les perceptions sont individuelles, les représentations sociales sont collectives ; elles sont partagées par un groupe de personnes. Les représentations sociales sont étudiées à travers le prisme des opinions, des croyances et des valeurs des acteurs sociaux.

\section{Représentations sociales suivant les opinions des communautés locales}

Les représentations sociales de la forêt suivant les opinions des communautés locales sont multiples. Celles qui sont jugées les plus pertinentes sont exposées. Elles sont relatives à l'utilité de la forêt, à la sécurité en zone forestière et à la représentation sociale de l'argent dans l'aménagement forestier qui sont des termes issus de l'agrégation de certaines perceptions sociales. Elles sont analysées suivant l'approche théorique des principes organisateurs de Doise (1990). 


\section{Utilité de la forêt}

$\mathrm{Du}$ fait de la dégradation avancée des forêts classées due à l'intervention anthropique, les communautés locales ont une représentation différenciée de ces aires protégées. Elles pensent que la forêt ne joue plus son rôle et qu'il est nécessaire d'opérer des réaffectations de la zone suivant l'utilité de celle-ci. Un habitant de Biguina 1 déclare à cet effet : " les gens ont attribué le nom forêt classée seulement. On dit forêt classée, forêt classée. On n'en voit plus l'utilité et les gens réclament la forêt, de leur donner ils vont cultiver dedans. Ça serait mieux», homme, 35 ans, Biguinal, le 03/09/2018. En effet, la revendication de plus en plus affirmée du déclassement de la forêt est largement partagée par les communautés riveraines. La majorité de la population étant dans le secteur agricole et compte tenu de la pression démographique, les terres cultivables s'amenuisent et deviennent moins fertiles. Pour satisfaire ce besoin d'étendre les superficies cultivables certains individus défrichent déjà illégalement des étendues de forêts surtout en périphérie de la forêt classée de Wari-Maro. La revendication de déclassement de la forêt répond à une logique de légitimation et de légalisation de ce qui se fait déjà de plus en plus dans la forêt. Le fait que les communautés locales se sentent impuissantes par rapport à la dégradation des forêts les incite à donc demander qu'une partie au moins de la forêt soit déclassée afin de leur permettre d'étendre leurs emblavures. La représentation sociale suivant laquelle "la forêt contient des ressources qui vont satisfaire les besoins des populations" trouve alors son sens.

\subsubsection{Sécurité en zone forestière}

L'insécurité dans la zone forestière a été évoquée comme une conséquence de la disparition de la brigade civile composée des chasseurs. En effet, les chasseurs de la zone de recherche s'étaient organisés en une brigade civile pour veiller à la sécurité des paisibles populations d'une part et d'autre part, pour subvenir à leurs besoins quotidiens en raison de la raréfaction du gibier. Seulement, les réformes intervenues dans le secteur de la sécurité ont fait disparaître ces brigades civiles. On assiste à une recrudescence des braquages, des prises d'otages, des viols et de violences physiques de tous genres. Un secrétaire d'un Comité Villageois pour le Développement Durable (CVDD) a relaté la situation en ces termes : " c'est dans la forêt classée là qu'il y a des braqueurs. C'est là leur refuge. Même avant-hier là, ils ont attrapé un jeune là, ils l'ont attaché, ils l'ont pris en otage et ils ont demandé aux parents d'envoyer de l'argent. (Extrait d'un entretien effectué à Alafiarou le 01/09/2018)

Les enlèvements de paisibles populations sont devenus un phénomène quasi quotidien dans la zone. Les auteurs de ces braquages et 
prises d'otages sont généralement des peulhs de nationalité étrangère selon les commentaires recueillis lors des entretiens et focus group.

«En plus de détruire la forêt, ils font maintenant enlèvement des gens, les peulhs qui sont dedans là, ils sont venus du Nigeria et du Niger; même les peulhs du Nigeria là ils font enlèvement des Peulhs autochtones, de Bariba, et ils disent que si vous ne donnez pas cinq millions, ils ne vont pas laisser votre homme si non ils vont le tuer; dans la forêt de Wari-Maro là. C'est ça qui effraie tout le monde. C'est l'insécurité totale; notez-ça» Un ancien élu local de Bétérou, le 01/09/2018

Les agents des forces de sécurité affectés dans la zone ne se montrent ni compétents, ni coopératifs par rapport à la prise en charge du phénomène selon les enquêtés. Ainsi, "Même les gendarmes qui sont à côté-là, s'il y a quelque chose, tu vas les appeler, ils ne vont même pas venir. C'est les chasseurs là seuls qui rentrent dans la forêt", membre de CVDD à Alafiarou le 01/09/2018. Les communautés riveraines se représentent alors la forêt comme une zone d'insécurité.

\section{Représentations de l'argent dans l'aménagement forestier}

Pour amener les communautés riveraines des forêts classées à adhérer à l'aménagement des forêts classées, il faut les motiver. Ainsi, la signature de contrats de cogestion dans lesquels mention est clairement faite des droits et responsabilités de chaque partie est souhaitée par les enquêtés qui évoquent l'approche adoptée par le PAMF : "Les gens recevaient des contrats. Les comités sous-traitaient les villageois et chacun avait son compte. Donc il faut faire des contrats avec eux pour qu'ils sachent qu'ils ont clairement quelque chose à gagner à une certaine échéance, TAOP, Manigri, 02/09/2018

Les moyens financiers, la rémunération, bref, l'argent constitue un facteur presqu'incontournable dans la gestion efficace des forêts classées des Monts Kouffé et de Wari-Maro dans la mesure où, délaissant leurs activités quotidiennes pour les activités de protection, de surveillance des forêts classées des Monts Kouffé et de Wari-Maro, les communautés riveraines doivent vivre de quelque chose. En compensation, il est donc nécessaire de rémunérer ces populations pour leurs efforts. L'absence de cette compensation constitue un facteur de démotivation pour ces populations engagées dans la gestion durable des forêts classées :

«Ah! Vous travaillez et on ne vous donne pas de l'argent. Mais quand on nous appelle pour aller en forêt, chaque fois, on nous appelle pour venir signer mais ils ne nous rappellent pas pour nous payer. Vous allez mettre de l'essence dans votre moto pour aller jusqu'à Tchaourou et revenir bredouille. Parfois, vous faites 
deux jours à les guider dans la forêt et finalement vous n'avez rien. Qui peux-tu aller trouver en brousse et parler avec afin qu'il te guide ? ", Chasseur, membre d'un CVDD lors d'un focus group à Wari-Maro le 31/08/2018

L'argent, le gain matériel doit-il être celui qui guide les actions des communautés riveraines à la gestion des forêts classées ? Cette question a été posée à des personnes ressources qui ont justifié son usage actuel. Quelques réactions seront exposées pour rendre compte des perceptions que les communautés riveraines ont de l'argent dans l'aménagement forestier. «Avant, avec quoi nos pères allaient en forêt? À pieds. Aujourd'hui, regarde la moto qui est garée là-bas. Elle coûte combien? Plus de trois cent mille francs CFA. Si tu dois labourer un champ sans avoir volé quelque chose ou détourné est ce que tu peux te l'acheter? C'est trop d'ambition et de convoitise qui font cela. Maintenant, c'est ça, le mensonge, la fourberie, c'est ça qui nous guide. Même moi qui vous parle, si je trouve une autre issue, vous pensez que je vais me souvenir de tout ce qu'on a dit ici aujourd'hui ? (rires aux éclats) », un élu local à Wari-Maro, le 31/08/2018

"Si les gens viennent te donner plusieurs fois cent mille, tu vas chercher à gagner plus non? ", Exploitant forestier, Wari-Maro le 31/08/2018

« Tantie, même nous qui parlons ici, c'est les gens qui sont venus nous montrer le n'importe quoi là. La personne vient et elle te dit: «ah, c'est sur telle richesse que toi tu dors comme ça?» l'argent que tu ne comptais pas gagner en un an, on te le remet en moins d'un mois. Tu vas faire comment? Il te dit: si tu me trouves telle espèce, tu verras ce que je vais te faire. C'est comme ça! Il n'y a même plus la vérité. C'est le nœud de notre objectif de travail. C'est ce que lui, il a fait pour trouver cette moto. Et moi, je me tais, je ne fais rien ? Si tu trouves les moyens de le faire, tu vas le faire aussi. C'est d'autres personnes qui sont venues nous ouvrir les yeux et tout gâter en fait », un élu local à Wari-Maro, le 31/08/2018

La question de la motivation se trouve également dans l'engagement de chaque partie à jouer avec efficacité sa partition. Les forestiers devraient agir dans le sens de donner plus de valeur à l'appui que leur apportent les communautés riveraines. En effet, parfois, ces communautés sentent leurs efforts non valorisées car disent-elles "Il n'y a pas de loi qui nous protège et qui nous permette de prendre des initiatives. Avant, lorsque nous arrêtons quelqu'un en infraction et que nous appelons les forestiers en renfort, 
parfois, ils nous demandaient de l'amener à Bassila ou à Bétérou », Chasseur, membre d'un CVDD à Wari-Maro

\section{Représentations sociales des forêts suivant les croyances des communautés riveraines}

Les représentations sociales de la forêt suivant les croyances des communautés locales sont présentées suivant la propriété de la forêt et la question de la durabilité.

\subsubsection{Propriété de la forêt}

Les communautés riveraines des forêts classées des Monts Kouffé et de Wari-Maro, suivant leurs groupes socioculturels d'appartenance, ont une certaine représentation de la propriété des terres abritant les forêts. Si les Peulhs pensent que la forêt appartient à Dieu, les autres communautés autochtones se représentent les forêts comme une terre appartenant à l'Etat. Ces enquêtés des autres groupes socioculturels (Nagot, Baatombu et Lokpa, propriétaires terriens), pensent que les peulhs ont cette représentation des terres forestières parce que : "En fait, ils ne savent pas où aller. C'est pour cela qu'ils disent cela. Avant, ce n'était pas comme ça non ?", ancien exploitant forestier, membre du CVDD de Wari-Maro le 31/08/2018. Ces propos sont à nuancer et à appréhender comme des prises de positions par rapport à des enjeux communs aux propriétaires terriens.

Les membres de la communauté peulh pratiquent l'élevage bovin et font subir à la forêt, un surpâturage depuis quelques années. Le fait que les membres de cette communauté d'éleveurs pensent que la forêt appartient à Dieu les autorise à se rendre dans cet espace sans limitation à la recherche d'eau et de pâturage; le terroir riverain étant saturé par les activités agricoles.

Les communautés nagot, baatonu, lokpa, propriétaires terriens et agriculteurs n'arrivent pas à freiner les peulhs dans leur élan, considérant leur caractère belliqueux ainsi que l'accompagnement insuffisant des autorités en charge de la gestion des forêts. Elles pensent donc que «Les peulhs profitent mieux de la forêt que nous les agriculteurs, les autochtones puisque eux, ils sont venus d'ailleurs. Or, ils ne nous donnent rien", pépiniériste, Wari-Maro, le 31/01/2018

En outre, la propriété de la forêt n'est pas contestable dans la conscience collective car les communautés locales estiment que leurs terres ont été cédées aux blancs (les colonisateurs) par le biais d'un marché de dupe. Ce marché était basé sur de la tromperie. L'expression baatonu "ba wii busi kwa" rend compte de cette réalité. Elle veut littéralement dire "ils l'ont roublé". Le roi avait été roublé par les blancs. Ces derniers, par la ruse, se sont approprié les terres de leurs ancêtres en leur miroitant des objets sans 
importance réelle. Le pays étant devenu indépendant dans les années 60 , ces forêts classées ont été transférées à l'Etat béninois. La transaction dolosive entre le pouvoir colonial et le pouvoir traditionnel a été évoquée par les enquêtés:

«En ce moment nos yeux n'étaient pas ouverts. C'est pour cela qu'on a cédé la forêt comme cela. En ce moment, les bouteilles, c'était quelque chose comme du miracle, quoi. Donc c'est les bouteilles que les blancs ont amené pour trahir ici. En ce moment, si vous avez de miroir dans votre chambre, vous êtes riche. On vient, on te donne miroir. Toi, tu peux donner tout ce que tu as comme biens (...). On ne peut pas dire que c'est arraché. C'est vendu mais la manière dont ils ont acheté là... ils amènent de vielles choses pour prendre les richesses. Ces choses ne sont même pas utiles pour nous maintenant. Or la terre était le bien que chacun possédait. Qu'est-ce qu'on peut dire? C'est la personne qui a sa chose et qui l'a vendue. La terre appartenait au roi et il l'a vendue. Est-ce que même jusqu'à présent les gens ne vendent pas les terres? Il y en a plein ». Secrétaire d'une CVC, Wari-Maro le 31/08/2018.

Ces étendues de terre, dans la conception des communautés locales étaient des espaces assez vastes parce que les ancêtres n'étaient pas nombreux. "Donc, ils ne savaient pas que le monde allait se peupler comme ça. Mais comme ils n'étaient pas nombreux, la terre était suffisante, vaste », secrétaire d'une CVC, Wari-Maro, le 31/08/2018. Il n'y avait donc pas de pressions sur les ressources forestières. Cette représentation de terres suffisantes pour tous ne dérangeait pas autrefois les habitants de la localité qui n'ont pas su planifier pour l'avenir. Les germes de la non-durabilité de la gestion des forêts se trouvaient aussi dans cette conception.

\section{Durabilité vue par les communautés riveraines}

La gestion des ressources naturelles incombait aux communautés locales avant la colonisation. Cependant, avec l'avènement du pouvoir colonial cette gestion a changé de mains. D'une gestion répressive et exclusive, les acteurs sont passés à une cogestion conformément aux nouvelles dispositions législatives (loi 93-009 du 2 juillet 1993 portant régime des forêts en République du Bénin).

Traditionnellement, les premiers gestionnaires des forêts sont les chasseurs ayant à leur tête, un chef chasseur. Il s'agissait d'une gestion spirituelle qui avait une certaine efficacité. Cette organisation traditionnelle a été redynamisée par le PAMF qui en a fait des confréries villageoises de chasse (CVC). Les investigations menées montrent que les ressources fauniques surtout dans la zone des Monts Kouffé et de Wari-Maro 
abondaient. Ainsi, la pratique des chasseurs à cette époque était de brandir les "trophées de chasse". Comme l'affirme l'un des interviewés :

« La CVC ne cherchait pas à gérer durablement la forêt. Elle ne sensibilisait pas à la protection de la forêt mais son rôle est de gérer les conflits internes entre chasseurs. Les chasseurs voulaient brandir des trophées de chasse. On se contentait de dire: "j'ai abattu tel nombre d'animaux". C'est comme quelqu'un qui va boire dans un bar sans contrôler sa consommation. Les chasseurs sont si puissants qu'ils arrivaient à détecter l'infidélité de leur femme. L'un des indices c'est que le chasseur ne tue pas d'animal quand il va à la chasse. Là, il procède à la consultation traditionnelle avec fusils. Les parents n'avaient pas cette idée de gestion durable. La forêt sacrée est différente de la forêt classée ». Un TAOP de la zone de WariMaro, Parakou le 31/08/2018.

Cette pratique n'allait pas dans le sens de la pérennisation des ressources. La perception trompe-l'œil de l'abondance des animaux donnait la sensation aux chasseurs que ces ressources n'allaient jamais tarir. C'est sans doute l'une des raisons pour lesquelles le PAMF a choisi de travailler avec ces CVC. L'intention première étant de les utiliser dans la protection des ressources naturelles de la forêt classée. Ainsi, "Pour mieux garder la viande qu'on est en train de fumer, le meilleur gardien est le chien. Dès qu'on responsabilise le voleur, il n'y a plus de vol ». Un TAOP de la zone de Wari-Maro, Parakou le 31/08/2018.

\section{Discussion}

Les communautés riveraines des forêts classées des Monts Kouffé et de Wari-Maro adoptent des pratiques vis-à-vis de leur environnement en raison des représentations sociales qu'elles en ont. Moscovici (1984) pense dans ce sens que les représentations sociales interviennent dans l'orientation des conduites des rapports sociaux (inter personnel et inter groupe). Pour comprendre les rapports qui lient les communautés à la forêt, il convient de revenir sur leur relation avec la nature, d'examiner la place attribuée à la forêt par la culture (Abanda Ngono, 2017). Les communautés riveraines des forêts classées des Monts Kouffé et de Wari-Maro ont des liens économiques, spirituels et politiques avec les forêts classées avoisinantes dictées par les représentations qu'elles ont de leur milieu de vie. Ainsi, en matière d'usages économiques, plusieurs pressions sont exercées sur les ressources forestières. Il s'agit des pressions agricole, pastorale, de coupe, de chasse et de pêche. Suivant ces comportements, il est nécessaire de documenter les représentations de l'espace forestier par ces populations et 
ensuite de confronter ces comportements à ceux que peuvent avoir d'autres populations à ce propos.

Les pressions agricoles exercées sur le milieu sont dictées par les caractéristiques des groupes socioculturels qui peuplent le milieu. Ce sont en majorité des cultivateurs issus des groupes socioculturels Nagot, Adja, Holli, Wama, Ditamari ayant migré vers la région à la recherche de terres fertiles. On peut donc en déduire que la pratique extensive de l'agriculture est motivée par le fait que les terres de la région sont très fertiles et que les peuples qui exercent cette activité ont vocation à le faire. L'espace réservé à l'agriculture dans le terroir villageois étant devenu insuffisant, l'espace forestier est pris d'assaut par les cultivateurs qui savent qu'ils sont en infraction et qui, pour cette raison, ont posé comme doléance le déclassement d'une partie de la forêt pour des fins agricoles. Pour le moment, quelques champs sont érigés à la périphérie de la forêt classée de Wari-Maro. Ainsi, selon l'approche théorique des principes organisateurs de Doise (1990) dans l'étude des représentations sociales, les interactions entre les acteurs sociaux se construisent dans un équilibre à l'intérieur des rapports de communication. Ainsi, lorsque ces interactions se construisent autour des problèmes investis d'une forte signification, il se produit des prises de positions individuelles (représentations/perceptions individuelles) liées à l'appartenance sociale. Ainsi, les variations observées d'un individu à un autre doivent être considérées comme des prises de position par rapport à des enjeux communs. Cette doléance se justifie parfaitement par rapport à cette théorie puisque l'enjeu agricole est commun aux groupes socioculturels qui pratiquent cette activité. Une étude de la FAO (2016) estime que les pertes de superficie forestière sont d'autant plus importantes dans les pays à faible revenu que les investissements dans l'agriculture et les forêts sont modestes. L'étude a montré l'importance d'un aménagement intégré du territoire et d'approches participatives faisant appel à des outils tels que les évaluations de l'aptitude des terres et de la prise en compte pleine et entière des vues des parties prenantes ; ceci, dans le but d'améliorer la gouvernance des terres.

Les villages à pression de coupe sont des villages à partir desquels les incursions dans la forêt classée sont organisées pour le prélèvement de bois d'œuvre ou pour la carbonisation (Tomety, 2002). Ces villages sont des points importants de ravitaillement en madriers et / ou en charbon de bois. Les pressions de chasse sont également courantes dans la zone de recherche. En cette matière, chaque village riverain a droit à un territoire de chasse dans les forêts classées des Monts Kouffé et de Wari-Maro (PAMF, 2006). Il en est de même de la pression pastorale qui prend de plus de l'ampleur surtout avec l'exacerbation du phénomène de transhumance transfrontalière. A ce 
propos, les gouvernants béninois ont pris des mesures pour interdire le phénomène ${ }^{1}$.

Selon Greffet (2011), ce sont les caractéristiques socio-économiques qui influencent le plus l'adoption de gestes concrets, les « bonnes "pratiques étant plus répandues parmi les ménages aisés. Les communautés locales se représentent donc les forêts selon leur utilité. En effet, pour les enquêtés, la forêt devrait être utile pour les populations riveraines L'analyse des représentations économiques que se font les populations riveraines de la forêt permet de mieux comprendre les pressions qui sont exercées sur les forêts classées des Monts Kouffé et de Wari-Maro et cela explique aussi en partie l'insistance des populations à faire déclasser une partie de la forêt afin que les cultivateurs y pratiquent l'agriculture. Cette insistance peut également répondre à une logique de légalisation et de légitimation de la pratique déjà en cours. Ainsi, les perceptions «la forêt classée améliore la vie des populations », la « forêt classée est une terre arrachée aux ancêtres », «la forêt classée appartient à tout le monde » sont de nature à encourager l'exploitation non-durable des forêts classées des Monts Kouffé et de WariMaro. Le fait que les cultivateurs, les exploitants forestiers, les chasseurs, les peulhs pasteurs et les pêcheurs investissent l'espace forestier en totale ignorance du PAP élaboré pourrait se justifier par ces perceptions sociales. «Sachant que les populations rurales africaines dépendent directement de l'environnement, l'intérêt et les représentations locales vis-à-vis de celui-ci sont souvent d'ordre pratique et technique : il s'agit de le travailler afin de survivre » (Fairhead et Leach, 1994). Ces auteurs vont plus loin en estimant que la compréhension des représentations sociales que se font des individus des ressources naturelles, est indispensable à l'appréhension de leurs modes de gestion. On pourrait alors déduire que les concepts techniques utilisés pour décrire et maîtriser les processus écologiques affectant les sols, l'eau, la végétation et les animaux sont spécifiques à la culture d'un peuple donné. Ainsi, les acteurs sociaux ne se contentent pas d'interpréter ou de s'adapter à leur environnement externe mais ils le façonnent tout au long de leur existence d'où la nécessité d'interroger ces représentations et pratiques par groupe socioculturel. Aussi, en plus de fournir des ressources matérielles et culturelles importantes pour la survie, la forêt est également un abri, un habitat pour plusieurs communautés dans le monde. Son état écologique détermine la production agricole, la pêche et la chasse, principales activités de subsistance des communautés forestières. La forêt est donc également un capital territorial (Gilbert, 2013).

${ }^{1}$ Juridiquement, une loi supranationale, notamment celle de la CEDEAO qui régit le phénomène a la primeur sur les lois nationales. On pourrait donc s'interroger sur cette décision nationale du Bénin de contredire une décision supranationale à moins de ne plus être parti au traité de la CEDEAO. 
Les communautés locales entretiennent des relations spirituelles avec les forêts classées des Monts Kouffé et de Wari-Maro. En effet, la forêt abrite les divinités et autres ancêtres auxquels les communautés consacrent périodiquement des cultes. Comme l'a affirmé Ouassa Kouaro (2014), la forêt constitue une source de symboles mythico-religieux et l'habitat des esprits qu'ils soient bons ou mauvais. La forêt permet ainsi à ces groupes sociaux d'entrer en relation avec le surnaturel. Or, la plupart des concepteurs des programmes de gestion des forêts ne perçoivent généralement de la forêt que la fonctionnalité et la nécessité d'une utilisation rationnelle de ses ressources notamment le bois et les ressources fauniques. Ils négligent souvent les dimensions symboliques qui influent sur les rapports des populations avec la nature environnante (Diop, 2012). La forêt a toujours été perçue dans sa dimension biologique, économique et environnementale. Pourtant, comme environnement humain, la forêt est d'abord un élément d'intégration de l'espace, de l'identité et de la culture. Cette dimension culturelle et anthropologique de la forêt est un préalable important pour comprendre la réalité du territoire forestier (Abanda Ngono, 2017). La compréhension des réalités du territoire sont donc très importantes dans l'élaboration des plans d'aménagement participatifs des forêts. En effet, selon cet auteur, la forêt représente pour des populations plus que l'étendue boisée que perçoit le sens commun. Elle traduit une réalité beaucoup plus complexe qui n'est souvent perceptible que dans les représentations qu'elles se font de cet espace. Les dimensions symboliques des forêts sont alors des variables incontournables dans la gestion durable des forêts puisque le territoire considéré comme sacré pour la population peut être épargné par l'exploitation anarchique. (Arouna et al, 2017)

Les communautés locales ont également des relations politiques avec les forêts classées. En effet, les politiques forestières ainsi que les personnalités politiques influencent le vécu des communautés riveraines des forêts classées étudiées. S'il est admis par Eisein et al (2015) que « le régime foncier forestier dominant, dans lequel l'Etat détient un contrôle absolu des ressources forestières, n'est pas parvenu à gérer durablement les forêts, réduire la pauvreté ou améliorer les moyens d'existence », la nécessité de la gestion participative des forêts tropicales n'est plus à démontrer. La gestion participative des ressources forestières fait apparaitre des questions de gouvernance. Ces auteurs dépeignent des situations où les taxes forestières sont sous-estimées par rapport à la valeur des ressources exploitées. Ce qui signifie que des ressources et même des projets sont détournés au détriment des Etats (par corruption) des fonctionnaires. Cette situation est due au pouvoir que représente l'argent dans toute œuvre humaine notamment dans l'aménagement forestier. En effet, Capozza et al (1995), dans une étude sur les représentations sociales de l'argent sont parvenus à démontrer que : le 
noyau central de la représentation est le binôme argent-pouvoir. Ils ont également trouvé qu'il y a incohérence entre les évaluations fournies au niveau de l'échelle de similarité et les réactions émotionnelles que l'argent produit. Cette incohérence indique la complexité sémantique du concept. Le manque d'espace géographique disponible pour les activités économiques diverses aggravé par une volonté politique et une capacité institutionnelle faibles ne sont pas de nature à assurer une durabilité sociale de l'aménagement forestier.

La mise en place des comités de cogestion a nécessité le choix de représentants des différentes couches de la population. Eisen et al. (2015) pensent à ce propos que le processus de mise en place de ces structures "omet de préciser comment ceux-ci seront nommés ou comment leur relation à la forêt sera prouvée (...). Il se peut qu'une autorité politique soit conférée à des chefs non représentatifs, qui agissent ou non en fonction de certains intérêts, ou à des membres en collusion avec des fonctionnaires corrompus, dont le rôle est d'approuver lesdits fonctionnaires. ». La représentativité des membres des comités, quelque fois politisée pose le problème de légitimité de ces membres et par ricochet de ces organisations, et donc de leur efficacité sur le terrain. Cette problématique de la légitimité des membres des comités a également été abordée par Baguiri (2016) et il importe d'y veiller pour la durabilité sociale des actions entreprises en vue de la mise en œuvre efficiente du plan d'aménagement des forêts classées des Monts Kouffé et de Wari-Maro. Le plan d'aménagement est l'instrument de la durabilité de la gestion forestière. Les analyses effectuées prouvent la durabilité sociale de l'aménagement forestier dépend des représentations sociales des communautés. L'hypothèse posée est donc vérifiée.

\section{Conclusion}

Les communautés locales des forêts classées des Monts Kouffé et de Wari-Maro sont des peuples qui entretiennent une relation étroite avec leur milieu de vie. Cet environnement qu'est la forêt joue un rôle important dans la survie physique mais aussi spirituelle et politique de l'homme. Ainsi, les comportements adoptés par les membres de ces communautés vis-à-vis de ces forêts sont d'abord économiques puisque la majorité des acteurs vivent des ressources de leur milieu. Elles exercent plusieurs pressions sur le milieu forestier. Ainsi, la pression agricole, la pression de chasse, de pêche, de coupe sont dictées par un souci de survie mais aussi par une volonté d'avoir de meilleures conditions de vie. Ce qui laisse transparaitre que les représentations et perceptions économiques des forêts jouent un rôle prépondérant quant à la gestion durable des aménagements forestiers. Les forêts ont plusieurs connotations pour les communautés riveraines des forêts classées des Monts Kouffé et de Wari-Maro. Elles sont entre autres 
considérées comme un patrimoine de l'Etat quoiqu'elles se sentent spoliées et victimes d'un marché de dupe dans la transaction ayant transféré ce bien au blanc puis à l'Etat colonial et enfin à l'Etat moderne. Les forêts sont aussi considérées comme des espaces qui peuvent permettre aux communautés locales d'améliorer leurs conditions de vie même si de plus en plus, elles tendent à devenir une zone d'insécurité. On comprend alors aisément pourquoi en dépit de tous les efforts déployés pour garantir une gestion durable des espaces forestiers, les projets forestiers n'arrivent pas à garantir une durabilité sociale, condition sine qua none de la durabilité écologique de leur intervention. La durabilité sociale de l'aménagement des forêts classées des Monts Kouffé et de Wari-Maro dépend donc des représentations sociales des forêts

\section{References:}

1. Abanda Ngono, F. (2017). La forêt, un patrimoine commun de l'humanité ? Réflexion sur les conflits de représentations de la forêt à l'aune de la patrimonialisation, Ethique publique, 19 (2), 17 p.

2. Abric, J. C. (1989). L'étude expérimentale des représentations sociales. Dans D. Jodelet (dir), Les représentations sociales. pp.187203. Paris: Puf.

3. Abric, J. C. (1992). Système central, système périphérique: Leurs fonctions et leur rôle dans la dynamique des représentations sociales. Communication à la Première Conférence Internationale sur les Représentations Sociales, Ravello, Italie.

4. Arouna, O., Toko Imorou, I., Gibigaye, M., Alle, P., Tente, B. (2017). Analyse comparative de l'état de conservation des forêts classées, des forêts communautaires et des forêts sacrées au SudBénin (Afrique de l'Ouest) International Journal of Innovation and Applied Studies, 19 (1), 27p.

5. Baguiri, O (2016). Processus d'élaboration et de mise en œuvre du plan d'aménagement des forêts classées des Monts Kouffé et de Wari-Maro : enjeux et logiques des acteurs [Mémoire de DEA, EDP, Université d'Abomey-Calavi]. 140 p.

6. Caillaud, S. (2010). Représentations sociales et significations des pratiques écologiques : Perspectives de recherche, VertigO - la revue électronique en sciences de l'environnement [Online], 10 (2), 20p. URL : http://vertigo.revues.org/9881 ; DOI : 10.4000/vertigo.9881.

7. Capozza, D., Robusto, E., Squarza, R., De Carlo, N.A., (1995). Représentation sociale de l'argent, Papers on social Representation, Université de Padoue, Italy 4 (1), 21 p.

8. Diop M, Sambou, B et Ly, B. (2012). Représentations de la forêt et répercussions sur la gestion des ressources forestières au Sénégal, 
VertigO-la revue électronique en sciences de l'environnement 12 (2), 13p. DOI : 10.4000/vertigo.12319

9. Djogbenou, C.P., Glèlè Kakaï, R.,. Arouna, O. et. Sinsin, B. (2011). Analyse des perceptions locales des aménagements forestiers participatifs au Bénin, VertigO - la revue électronique en sciences de l'environnement, 11 (1). 18p .URL: http://vertigo.revues.org/10893; DOI: $10.4000 /$ vertigo.10893.

10. Doise, W. (1990). Les représentations sociales. Dans R. Ghiglione, C. Bonnet et J-F. Richard (Eds), Traité de psychologie cognitive, 3., pp.111-176. Paris : Dunod

11. Eisen, J., Counsell, S., Thornberry, F. (2015). Repenser la gestion communautaire des forêts $d u$ bassin $d u$ Congo. Rainforest Foundation, U K, 61 p.

12. Fairhead J et Leach M. (1994). Représentations culturelles africaines et gestion de l'environnement. Politique africaine, (53), pp.11-24.

13. FAO (2016). Situation des forêts du monde. Forêts et agriculture: défis et possibilités concernant l'utilisation des terres. Rome, $137 \mathrm{p}$

14. Gbedahi, O.L., Biaou, S.S.H., Mama, A., Gouwakinnou, G.N., Yorou, N.S. (2019). Dynamique du couvert végétal à Bassila au Nord Bénin pendant et après la mise en œuvre d'un projet d'aménagement forestier. International journal of biological and chemical sciences, http://www.ifgdg.org

15. Gilbert, A (2013). Les territoires forestiers de l'Ouest du Québec, entre centre et périphérie. Dans G. Chiasson et É. Leclerc (dir.), La gouvernance locale des forêts publiques québécoises. Une avenue de développement des régions périphériques?, pp. 89-122, Presses de l'Université du Québec. Québec.

16. Greffet P (2011). Le niveau de vie, principal déterminant des pratiques environnementales, in perceptions sociales et pratiques environnementales des français de 1995 à 2011. La revue du CGDD (Commissariat Général au Développement durable), pp 39-50.

17. Jodelet, D. (1989). Les représentations sociales. Paris: Puf. 310 pages.

18. Lavigne Delville, P. (1998). Environnement, dynamiques sociales et interventions externes construire et gérer l'interface. Dans : G. Rossi, P. Lavigne Delville, D. Narbeburu, Sociétés rurales et environnement, pp. 381-394. Paris, GRET-Regards, Karthala.

19. Moscovici, S. (1984). The phenomenon of social representations. In R. Farr \& S. Moscovici (Eds.), Social representations. pp369. Cambridge: Cambridge University Press. 
20. Moscovici, S., \& Hewstone, M. (1983). Social representation and social explanation. In M. Hewstone (Ed.), Attribution Theory. pp 95125. Oxford: Blackwell's.

21. Navarro O. (2009). Représentations sociales de l'eau dans un contexte de conflits d'usage : le cas de la sierra Nevada de santa Marta, Colombie, Cahiers Internationaux de Psychologie Sociale, 81, 65-86.

22. Ouassa Kouaro, M. (2014). Représentations symboliques des Anii et Nagot des forêts dans le département de la Donga., ALAC (4) $16 \mathrm{p}$. ISSN : 2219-6633.

23. Tomety, S. N. (2002) Rapport de la mission de l'expert en sociologie au Projet Aménagement des Massifs Forestiers d'Agoua, des Monts Kouffé et de Wari-Maro- PAMF, $104 \mathrm{p}$.

24. PAMF (2006) Plan d'Aménagement Participatif du complexe des forêts classées de Wari-Maro et des Monts Kouffé Volume A (Partie descriptive) Durée 10 ans, 267 pages.

25. Rousseau, M.-H. (2008). L'acceptabilité sociale de l'aménagement forestier sur l'île d'Anticosti, un territoire à vocation faunique [Mémoire de maîtrise, Université Laval].81 pages.

26. Weber, M. (1995). Économie et société, 1. Paris, Plon [1921]. 